\title{
Protección de los derechos de propiedad intelectual en el APEC
}

I

\section{ntroducción}

La protección de los derechos de propiedad intelectual en el foro de Cooperación Económica de Asia Pacífico (APEC) se fundamenta en las consideraciones sobre el tema contenidas en el Acuerdo sobre los Aspectos de los Derechos de Propiedad Intelectual Relacionados con el Comercio (ADPIC). ${ }^{1}$ Ello se hizo explícito en la Agenda de Acción de Osaka, en la que se establecen los pasos a seguir para poner en operación la Declaración de Bogor. En esta agenda se instituye que las economías miembros del APEC protegerán los derechos de propiedad intelectual, para lo cual adoptarán los principios del ADPIC en el año 2000. ${ }^{2}$ Es decir, según la disposición de la Agenda de Acción de Osaka, todas las economías del APEC deberían reforzar sus sistemas nacionales de protección de los derechos de propiedad intelectual, de manera que estuvieran en posibilidad de poner totalmente en operación el ADPIC a partir de enero de 2000. A diferencia de lo que estipula este último, en el APEC no hubo plazos de gracia para las economías menos desarrolladas, a las que se les había concedido, en el marco de la Organización Mundial de Comercio ( $\mathrm{OMC}$ ), que tenían hasta el último día de 2005 para ajustar sus sistemas de protección de la propiedad intelectual, en forma tal que a partir del primer día de 2006 pusieran en operación todas las disposiciones del ADPIC.

Así, el objetivo primordial fue que las economías del APEC aseguraran una protección efectiva y adecuada (incluyendo la legislación, administración y reforzamiento) de los derechos de propiedad intelectual en la región Asia Pacífico, con base en los principios de trato nacional, nación más favorecida y transparen-

* Investigador del Departamento de Estudios del Pacífico de la Universidad de Guadalajara.

ORCID http://orcid.org/0000-0003-0344-6895 cia, tal como se establece en el ADPIC y otros acuerdos relacionados. ${ }^{3}$ Esto aun cuando en el APEC no existe un acuerdo que obligue explícitamente a cada uno de los miembros a establecer mecanismos efectivos y adecuados para la protección de los derechos de propiedad intelectual, por lo que sólo lo hacen de manera referenciada al ADPIC. Parecería que no es necesario un acuerdo obligatorio, puesto que la mayoría de los integrantes del APEC son miembros firmantes de la oMc; ${ }^{4}$ sin embargo, dado que el foro no contempla disposiciones obligatorias para sus miembros, la protección de los derechos de propiedad intelectual, en la práctica, queda en manos de otros organismos.

En este trabajo se abordarán las principales disposiciones del APEC en materia de protección de los derechos de propiedad intelectual. Aquí se analizarán los elementos que muestren la importancia que da el APEC a la protección de estos derechos. Daré seguimiento a las reuniones del Grupo de Expertos en Propiedad Intelectual del APEC (IPEG) ${ }^{5}$ con el fin de ir rescatando los principales aspectos sobre la materia. De este modo, se analizará hasta qué grado se ha puesto en operación el ADPIC y su influencia en la determinación del nivel de protección de los derechos de propiedad intelectual en el APEC.

\section{Propiedad intelectual en el APEC y el ADPIC}

La entrada en vigor del ADPIC a partir de enero de 1995, como parte fundamental del acuerdo por el que se estableció la oMc, llamó la atención del APEC sobre la protección de los derechos de propiedad intelectual en el marco del organismo, por lo que en noviembre de 1995, en la Agenda de Acción de Osaka, se reconoció la importancia del tema y se propuso que 
sus miembros deberían fortalecer sus sistemas nacionales de protección de estos derechos. Así, el objetivo específico de la agenda señala el establecimiento de una protección adecuada y efectiva por parte de las economías miembros, incluyendo las leyes, la administración y la observancia de los derechos de propiedad intelectual en la región, con base en los principios de trato nacional y transparencia establecidos en el ADPIC. Por esta razón, el Grupo de Trabajo, formado en 1996 para lograr el objetivo del APEC en esta materia, propuso lo siguiente:

1) Avanzar en el diálogo sobre las políticas de propiedad intelectual en el área.

2) Recolectar e intercambiar información sobre la situación actual de la protección de los derechos de propiedad intelectual y los sistemas administrativos.

3) Estudiar medidas para la aplicación efectiva de los derechos de propiedad intelectual.

4) La total entrada en vigor del ADPIC antes del año 2000.

En relación con el primer punto de la propuesta, se estableció que se llevarían a cabo reuniones periódicas entre representantes, tanto del sector público como del privado, de cada una de las economías miembros, de manera que se propiciara un conocimiento más uniforme de las principales disposiciones vigentes, lo que facilitaría el entendimiento del sistema imperante en ellas. Con respecto al segundo punto, se acordó que cada economía proporcionaría información sobre el marco legal y administrativo vigente, de modo que se conformara una base de información acerca de la jurisprudencia relevante en la materia. En lo que toca al tercer punto, el Grupo de Trabajo se comprometió a buscar mecanismos que condujeran paulatinamente al apego irrestricto a la protección de los derechos de propiedad intelectual, procurando disminuir la brecha entre las disposiciones y su aplicación efectiva y práctica. Finalmente, el mayor compromiso es el que se refiere a la entrada en vigor del ADPIC a partir del primer día del año 2000, en forma completa y en todas las economías miembros.

En 2000, a raíz del acuerdo de modificación del Plan de Acción Colectiva, también establecido en Osaka, en noviembre de 1995, se estipuló que a partir de esa fecha el plan incluiría los siguientes puntos:

a) Puesta en operación del ADPIC;

b) Aseguramiento de la concesión expedita de los derechos de propiedad intelectual;

c) Observancia efectiva de los derechos de propiedad intelectual;

d) Educación pública sobre propiedad intelectual; y

e) Cooperación en el APEC sobre propiedad intelectual. ${ }^{6}$

El establecimiento de esta agenda tenía que ver con la etapa inicial en que se encontraba el tema de la protección de los derechos de propiedad intelectual en el APEC, por lo que en las reuniones subsecuentes se fue modificando. Sin embargo, la mayoría de estas modificaciones no han sido de fondo y permanecen enunciadas de manera general. El punto que obligatoriamente ha tenido que cambiar es el de la entrada en vigor del ADPIC, una vez que se cumplió la fecha para su aplicación en el APEC. Así, a mayo de 2003 los objetivos del grupo de expertos son:

- Profundizar el diálogo en las políticas de propiedad intelectual.

- Recabar e intercambiar información sobre la situación de la protección de los derechos de propiedad intelectual y los sistemas administrativos en la materia.

- Estudiar las medidas para el reforzamiento efectivo de la protección de los derechos de propiedad intelectual.

- Poner en operación total el ADPIC.

- Facilitar la cooperación técnica para poner en operación el ADPIC. ${ }^{7}$ 
Desde 1998, en las reuniones del IPEG se reportan las actividades que lleva a cabo este grupo, sobre todo en el establecimiento de medidas de cooperación que posibiliten a los miembros del APEC lograr el objetivo de la puesta en operación del ADPIC a partir del año 2000. Para alcanzar este fin, el IPEG, como parte de la cooperación multilateral en el foro, extendió la asistencia técnica a las economías del APEC, por medio de la realización de estudios que contribuyeran a acelerar los procesos legislativos en éstas. Además, se plantearon propuestas de entrenamiento de personal en el área de la protección a la propiedad intelectual. ${ }^{8}$

\section{El APEC es un mosaico de economías muy} heterogéneas, por lo que resulta difícil describir y, aún más, analizar cada aspecto de sus políticas en este ámbito. No obstante, es posible señalar que los niveles de protección han aumentado sustancialmente, sobre todo en los países en desarrollo, considerando que los más desarrollados no necesitaron hacer ajustes significativos a sus sistemas nacionales de protección en la materia.

De lo anterior, da cuenta el presidente del IPEG durante su reunión de marzo de 2000 en el reporte sobre la puesta en operación del ADPIC. En dicho reporte establece que se ha notado un conocimiento creciente de la importancia de la explotación de los derechos de propiedad intelectual y de la manera adecuada para aprovechar los frutos de la investigación a fin de fomentar el desarrollo económico de los miembros del APEC, por lo que "muchas economías han ido más allá de sus obligaciones técnicas para demostrar su adhesión al ADPIC". ${ }^{9}$ Ello ha promovido positivamente los elementos del sistema que dan efecto a este acuerdo.

Ahora bien, hasta aquí todo parece indicar que las economías del APEC han emprendido el camino hacia la protección efectiva de estos derechos en forma voluntaria, y se han comprometido a más de lo que se podría esperar de acuerdo con sus compromisos adquiridos en los diferentes foros multilaterales. Sin embargo, habrá que tomar en cuenta que durante las negociaciones del ADPIC, Estados Unidos, con su poderío económico, amenazó con imponer sanciones comerciales sobre los Estados, en especial los países en desarrollo, que supuestamente hubieran infringido o carecieran de protección a los derechos de propiedad intelectual estadounidenses en su territorio. ${ }^{10}$

Así pues, Estados Unidos promulgó la Ley de Comercio de 1984 (bajo la sección 301 de la Ley de Comercio de 1974), la cual establece que el presidente de Estados Unidos puede imponer sanciones comerciales a los países que, según su propia evaluación, no provean de protección a los derechos de propiedad intelectual de las empresas y ciudadanos estadounidenses. Además, en 1988 este país promulgó la Ley General de Comercio y Competitividad con el fin de reforzar aún más la protección de estos derechos en los mercados externos. Una de las principales disposiciones de esta ley, la "Especial 301", otorga mayores poderes al presidente para imponer sanciones económicas severas sobre los países que no hubieran tenido éxito al respecto. Dicha sección estipula que la falta de protección se considera una práctica de comercio ilícita. Así, al amparo de esta ley, Estados Unidos investigó las supuestas infracciones de las leyes 
sobre propiedad intelectual de éste en China, Brasil, India, Taiwan, Corea, México, Arabia Saudita y Tailandia (la mayoría de ellos son ahora miembros del APEC). ${ }^{11}$

Todas estas medidas ya no son necesarias a partir del compromiso en el APEC de la entrada en vigor del ADPIC, puesto que el funcionamiento del régimen internacional ${ }^{12}$ las sustituyó de manera efectiva. El régimen internacional, representado por el ADPIC, es en esencia lo mismo que las amenazas de represalias contra las políticas de los países en desarrollo, en el sentido de que actúa como la instancia que prohíbe que tal protección sea débil, por lo que el ADPIC funciona como un sustituto de las negociaciones bilaterales coercitivas por parte de los países desarrollados, sobre todo de Estados Unidos. ${ }^{13}$

Así, el funcionamiento del régimen internacional de protección de la propiedad intelectual en el APEC ha permitido, en general, un aumento en el nivel de protección de los países miembros y es clara la tendencia de que dichos niveles están en proceso de mejoramiento. Esto puede corroborarse en la declaración de los integrantes del IPEG en el sentido de que las 21 economías del foro completaron el mejoramiento legislativo conforme al ADPIC, según la verificación realizada sobre el estado de la puesta en operación del acuerdo. ${ }^{14}$

Ahora, si bien se puede argumentar que el aumento en el nivel de la protección se debe a la presión ejercida por los países desarrollados del foro en otras instancias, por ejemplo durante el proceso de negociación del ADPIC, también es cierto que el contenido y ritmo de las reformas hechas al respecto en los países menos desarrollados del APEC con frecuencia han ido más lejos de lo que los propios negociadores del ADPIC por parte de Estados Unidos, en particular, y de los demás países desarrollados pudieran haber esperado.

Otro factor que pudiera considerarse para explicar la adopción voluntaria del ADPIC es el surgimiento de industrias tecnológicas internas en algunas de las economías miem- bros. Es decir, el rápido crecimiento económico y el desarrollo tecnológico de algunos integrantes del APEC han determinado que éstos experimenten un interés creciente en la protección de los derechos de propiedad intelectual de sus propias obras e inventos, además de los del resto de las economías. No obstante, este argumento no explica porqué otras economías dentro del foro, que no registran los mismos niveles de crecimiento y desarrollo tecnológico, también están mejorando sus sistemas de protección.

El factor que se argumentará aquí como la principal explicación de esta mejora es el funcionamiento del régimen en la materia representado por el ADPIC. Aunque debe aclararse que, en general, durante los noventa el mundo se movió claramente hacia un sistema internacional de protección de los derechos de propiedad intelectual, lo que requirió la aplicación de estándares mínimos de protección más altos. Un componente de suma importancia en este cambio fue la adopción unilateral de leyes más fuertes y procedimientos de aplicación más confiables en numerosos países en desarrollo. Estas decisiones se tomaron, en parte, propiciadas por presiones externas, y por la percepción creciente de que un sistema de protección más fuerte cumple un papel importante para atraer inversión y tecnología. ${ }^{15}$

Este último factor parece ser el más importante en el caso del APEC. El tema de la protección a los derechos de propiedad intelectual aparece citado apenas en la Agenda de Acción de Osaka (1995), donde se establece el compromiso de adoptar el ADPIC a partir del año $2000 .{ }^{16}$ Antes de esa fecha el tema no se había contemplado en ninguno de los documentos importantes del APEC, por lo que tampoco se había creado, en su interior, alguna instancia que abordara el asunto. Así, fue hasta 1996 cuando se formó el Grupo de Trabajo sobre Propiedad Intelectual, el cual se consolidó como Grupo de Expertos del APEC en la materia (IPEG) a partir de 1998.

Este grupo, compuesto por representantes de cada una de las economías, se reúne dos 
veces al año, por lo que la interacción entre ellos es muy frecuente. También participan en diferentes reuniones internas de cada una de las economías del foro, lo que permite, además del intercambio de ideas entre sus homólogos, la difusión de sus conocimientos y propuestas en la materia entre las comunidades interesadas y el público en general. A este efecto, cada economía se compromete año con año a informar a los demás miembros de su programa de actividades, con lo que se posibilita ampliamente el seguimiento del proceso de difusión del tema, lo que a su vez facilita el avance de los objetivos compartidos en el ADPIC.

Toda esta serie de actividades compartidas y de múltiple contacto entre los responsables y estudiosos de la protección de los derechos de propiedad intelectual ha permitido que se puedan ir trazando metas comunes sin necesidad de forzar medidas impuestas de unas economías sobre otras. El IPEG se ha propuesto trabajar en proveer las bases que apoyen un sistema fuerte y efectivo de protección de tales derechos, para lo cual es necesario convencer a todas las economías de que un sistema con estas características es sumamente importante para el comercio, ya que brinda confianza a las empresas de que este tipo de derechos serán respetados y los beneficios llegarán a quien o quienes sean los verdaderos dueños.

El que en el APEC se presente esta situación es, en gran medida, producto de la evolución de las ideas y del conocimiento sobre el tema en la mayoría de los integrantes del foro. A lo anterior ha contribuido en forma sustancial el grupo de expertos, al establecer que las economías del APEC se pueden beneficiar mediante la divulgación de sus experiencias en esta área, y al promover un flujo de información productivo para avanzar en los objetivos comunes de los miembros del foro. Así, es evidente que las comunidades epistémicas ${ }^{17}$ son canales cruciales a través de los cuales circulan ideas nuevas desde la sociedad hacia los gobiernos, y de un país a otro. ${ }^{18}$
La difusión de ideas y conocimientos nuevos es una de las tareas primordiales del IPEG, tal como se estipula en sus objetivos. ${ }^{19}$ Mediante esta actividad los expertos pueden comunicar sus ideas a sus contrapartes, las cuales pueden a su vez influir en los gobiernos y verse reflejadas en las políticas de éstos. Asimismo, las negociaciones que los gobiernos emprendan es posible que se vean condicionadas o influidas por la información provista, en un principio, por los expertos, ya que el conocimiento proporcionado por el grupo no sólo ejerce una determinada influencia en los debates acerca del tema, sino que también puede ayudar a los Estados a identificar sus principales intereses en situaciones más particulares.

Sin duda, la participación y las actividades llevadas a cabo por el IPEG, han permitido un mayor entendimiento de los sistemas nacionales de propiedad intelectual y han ampliado la confianza hacia ellos. De otro modo, la falta de información precisa y pertinente acerca de tales sistemas, y dada la dificultad y complejidad para interpretarlos, puede dar pie a malentendidos, lo que a su vez pudiera conducir a fricciones bilaterales o multilaterales. ${ }^{20}$

En consecuencia, lo que ha permitido que el régimen de protección de derechos de propiedad intelectual, representado por el ADPIC, opere sin complicaciones sustanciales en el APEC es la asimilación que de dicho régimen se ha tenido en las economías miembro, donde el grupo de expertos en la materia ha tenido una participación importante, permitiendo que la evolución del conocimiento y las ideas nuevas lo fortalezcan. Así, el grupo de expertos ha defendido el régimen y propiciado su funcionamiento en el APEC, al considerarlo como el mejor mecanismo establecido para hacer frente a la problemática que originó su propia creación.

\section{Conclusiones}

En el APEC, el régimen internacional de protección de los derechos de propiedad intelec- 
tual ha adquirido su propio funcionamiento, de manera independiente a la evolución y fortaleza, o debilidad en todo caso, del régimen global representado por el ADPIC. En este foro, como vimos, el plazo para la entrada en vigor del ADPIC se ha adelantado para las economías menos desarrolladas, pues de tener la opción de acatar las disposiciones del ADPIC hasta principios de 2006, están "obligadas", en el marco y bajo las condiciones del APEC, a sujetarse al régimen a partir del año 2000. Esto indica cierta fortaleza del régimen en el APEC, quizá mayor a la del régimen global, ya que, a pesar de que las disposiciones y acuerdos en el APEC no son obligatorias sino voluntarias y no existen mecanismos de imposición ni medidas de represalia para quien no cumpla con lo acordado, se ha notado un avance sustancial en la aplicación de las medidas estipuladas, lo que ha incidido en un aumento del nivel de protección de los derechos de propiedad intelectual en la mayoría de las economías integrantes del foro.

Entre los factores que han permitido este aumento, el más importante es la existencia y funcionamiento del Grupo de Expertos en Propiedad Intelectual del APEC. Este grupo ha desempeñado su papel como una comunidad epistémica, la cual cumple con la tarea de fomentar la atención sobre el tema de la propiedad intelectual, propiciando que se le otorgue la importancia que merece, dada la relevancia que ha adquirido en las necesidades de crecimiento de los países, sobre todo para los que están en vías de desarrollo. Con ello, el IPEG ha permitido que el régimen internacional de propiedad intelectual, representado por el ADPIC, tenga una aplicación sustancial en la protección efectiva y adecuada de los derechos de propiedad intelectual en la región Asia Pacífico, específicamente en las economías del APEC.

\section{Notas}

1 También conocido como tRIPS, por sus siglas en inglés (Trade Related Aspects of Intellectual Property Rights).

2 The Osaka Action Agenda. Implementation of the Bogor Declaration, en http://www.apecsec.org.sg/virtualib/ history/osaka/agenda.html.

3 Ibid.
4 En el caso del APEC, sólo dos economías no han firmado su adhesión a la omc: Rusia y Vietnam.

5 Aunque el grupo de trabajo se conformó en 1996, el grupo de expertos empezó a funcionar como tal a partir de 1998.

6 Foro de Cooperación Económica Asia Pacífico (APEC), en http: / / www.impi.gob.mx/web/docs/relaciones / index relaciones.html.

7 Intellectual Property Rights (fechado: 27 de mayo de 2003), en http://www.apecsec.org.sg/committee/ intellectual upd.html.

8 Chair's report to APEC CTI on the Intellectual Property Rights Expert Group vI, en http://www.apecsec.or.sg/ download/virtualib/ipeg/ipr06-aus.exe.

9 APEC Intellectual Property Rights Expert Group X, en http: / / www.apecsec.or.sg/download/virtualib/ipeg/ IpegJpnChairMar.exe.

10 Huala Adolf, "Trade-related aspects of intellectual property rights and developing countries", The developing economies, xxxIx-1, marzo 2001, pp. 53-54.

11 Ibid., p. 54.

12 Este se entiende como los principios, normas, reglas y procedimientos para la toma de decisiones alrededor de los cuales las expectativas de los actores convergen en un área de tema dada de relaciones internacionales. Para una profundización sobre el concepto, véanse: Stephen D. Krasner (ed.), International regimes, Cornell University Press, Ithaca, 1983; y Andreas Hasenclever, Peter Mayer y Volker Rittberger, Theories of international regimes, Cambridge University Press, Cambridge, Reino Unido, 2000.

13 Hiroki Tohya, "TRIPS and policies of APEC on intellectual property rights: economic theory and political reality", Working Paper Series 96/97, núm. 8, IDE APEC Study Center, marzo de 1997.

14 Chair's paper on the implementation of the TRIPS Agreement (annex 2), en http://www.apecsec.or.sg/ download/virtualib/ipeg/IpegJpnChairMar.exe .

15 Keith E. Maskus, "The international regulation of intellectual property", Seminar Paper 97-11 del Centre for International Economic Studies, University of Adelaide, Australia, octubre de 1997.

16 Cfr. supra.

17 Las comunidades epistémicas se definen como redes de profesionales con experiencia y competencia reconocida en un dominio particular y una opinión autorizada en el conocimiento relevante para las políticas dentro de ese dominio o área del tema.

18 Andreas Hasenclever, Peter Mayer y Volker Rittberger, op. cit., p. 149.

19 What is APEC/IPEG?, en $h t t p: / / w w w . a p e c i p e g . o r g / w h a t i s /$ default.asp.

20 APEC Intellectual Property Rights Expert Group vIII, en http://www.apecsec.org.sg/download/virtualib/ ipeg fukuoko.exe. T:? 